\title{
Algoritmo BVNS Aplicado no Problema de Restauração de Redes de Distribuição de Energia Elétrica
}

\author{
Antonio M. Cossi ${ }^{1}$ \\ DMAT/FEIS-Unesp, Ilha Solteira, SP \\ Guilherme F. Afonso ${ }^{2}$ \\ SENAC, Campinas, SP
}

\begin{abstract}
Resumo. Neste trabalho, é proposto um modelo baseado na técnica de solução BVNS (Variable Neighborhood Search) para resolver o problema de restauração de sistemas de distribuição de energia elétrica (PRSDEE). O modelo matemático consiste em minimizar o custo de energia não distribuída no sistema devido às seções que não foram restabelecidas após o processo de restauração. Trata-se de um problema de programação não linear inteiro misto (PNLIM), no qual as propostas de solução são obtidas através do algoritmo BVNS. A estrutura de vizinhança do algoritmo BVNS é baseada na técnica conhecida como Representação Nó-Profundidade. Cada solução não factível, obtida pelo algoritmo BVNS, é penalizada. Para testar a eficiência do modelo proposto, são apresentados resultados de testes em um sistema de distribuição de 54 seções.
\end{abstract}

Palavras-chave. Restauração de Redes, Basic Variable Neighborhood Search, Representação NóProfundidade

\section{Introdução}

O problema de restauração de sistemas de distribuição de energia elétrica (PRSDEE) consiste em restabelecer o sistema energizando regiões da rede que ficaram sem energia elétrica devido a interrupções permanentes (faltas permanentes) na rede [7].

Neste trabalho, o PRSDEE consiste em restabelecer o sistema através de uma topologia de rede que restaure o maior número possível de seções após a interrupção no fornecimento de energia elétrica. O objetivo é minimizar o custo da energia não distribuída no sistema devido às seções que não foram restabelecidas após o processo de restauração. Trata-se de um problema de programação não linear inteiro misto (PNLIM), em que cada proposta de solução topológica da rede é obtida através do algoritmo BVNS (Basic Variable Neighborhood Search) [2]. A estrutura de vizinhança do BVNS é baseada na técnica RNP (Representação Nó-Profundidade) através da quantidade de podas na rede [5], alterando assim sua estrutura topológica. Além disso, o procedimento de busca local utilizado pelo algoritmo para promover a mudança na estrutura de vizinhança consiste em aplicar podas nas seções afetadas pelas faltas de energia utilizando um dos operadores da RNP. O funcionamento da técnica RNP, bem como de seus operadores podem ser estudados em [5]. Para resolver o PRSDEE utilizando o algoritmo BVNS, para cada solução do conjunto de vizinhança do algoritmo, caso alguma restrição seja violada, ou seja, não for factível, esta é panalizada.

O algoritmo BVNS é uma extensão do algoritmo VNS (Variable Neighborhood Search), que integra as características do algoritmo VND (Variable Neighborhood Descent), o qual permite

\footnotetext{
1 antonio.cossi@unesp.br

2 guilhermeflorindoafonso@gmail.com
} 
encontrar soluções ótimas de qualidade, e do algoritmo RVNS (Reduced Variable Neighborhood Search) que permite encontrar novas regiões promissoras a partir de um ótimo local, sendo estas outras duas extensões, também, do algoritmo VNS. Assim, o algoritmo BVNS é mais eficiente combinando a busca local com mudanças sistemáticas de estruturas de vizinhança em torno do ótimo local [2].

Na literatura encontram-se diversos trabalhos que utilizam diferentes técnicas de solução para resolver o PRSDEE. Dentre as propostas existentes, podemos destacar os trabalhos que utilizam os métodos heurísticos $[3,6]$ e metaheurísticos $[1,7]$, para a solução do problema. Dentre os trabalhos citados, destaca-se o trabalho desenvolvido por [7] que utiliza a metaheurística Tabu Search para resolver o PRSDEE, e cujo sistema de vizinhança é baseado na técnica RNP.

A contribuição deste trabalho, além do desenvolvimento de um modelo eficiente para resolver o PRSDEE, é avaliar a aplicação da metaheurística BVNS associada à técnica RNP para resolver este tipo de problema.

Para testar a eficiência do modelo proposto para o PRSDEE, apresentam-se resultados de um sistema de distribuição de média tensão contendo 54 seções de cargas, alimentadas por 3 subestações. Cada seção de carga é composta por um conjunto de consumidores. Os dados do sistema foram adaptados de [4].

\section{Modelo Matemático}

O PRSDEE é formulado como um problema de programação não linear inteiro misto (PNLIM), cuja função objetivo (1) procura minimizar o custo da energia não distribuída devido às seções que não foram restabelecidas pelo processo de restauração, conforme descrito a seguir.

$$
\operatorname{Min} C_{E N D}=\left(\sum_{k=1}^{N_{C D}} R_{C D} \cdot P_{C D_{k}}\right) \cdot Y_{C D_{k}}
$$

As restrições do problema, descritas pelas equações (2) a (6), são técnicas, operacionais e físicas do sistema de distribuição.

$$
\begin{gathered}
S_{s}^{\max }=\sum_{s=1}^{N_{s u b}} \cdot\left(\sum_{k=1}^{N_{s e c}} S_{C_{k}}^{s}+\sum_{k=1}^{N_{C D}} S_{C D_{k}}^{s}+\sum_{k=1}^{N_{l}} S_{P_{k m}}^{s}\right) \\
f_{k m} \leq f_{k m}^{\max } \\
V_{k}^{\text {min }} \leq V_{k} \leq V_{k}^{\max } \\
Y_{C D_{k}}=0 / 1 \\
\sum_{j=1}^{N l} j=\left(\sum_{k=1}^{N_{\text {sec }}} k\right)-1
\end{gathered}
$$

em que: $C_{E N D}$ é o custo total da energia não distribuída, para cada $\mathrm{kW}$ interrompido, devido às cargas que permaneceram desligadas após o processo de restauração; $R_{C D}$ é o valor da taxa, em $\$$, de remuneração individual por $\mathrm{kW}$ interrompido, devido às cargas que permaneceram desligadas após o processo de restauração; $P_{C D}$ é a demanda, em $\mathrm{kW}$, de cada seção afetada pela falta, exceto a própria seção onde ocorreu a falta; $Y_{C D}$ é a variável que indica se a seção foi restabelecida ou não (1 para seção não restabelecida e 0 para seção restabelecida); $N_{\text {sub }}$ é o total de subestações do sistema; $N_{C D}$ é o total de seções desligadas após a falta permanente, exceto a seção da falta; $S^{\max }$ é a demanda máxima que cada subestação pode suprir; $S_{C}$ é a demanda de cada seção do sistema, exceto as seções afetadas pela falta permanente e a seção do defeito; $S_{C D}$ é a demanda das seções 
restauradas; $S_{P}$ é demanda das seções restauradas; $N$ sec é o total de seções do sistema, exceto as seções afetadas pela falta e as próprias seções onde ocorreram as faltas; $N_{l}$ é o total de ramais do sistema; $f$ é o fluxo de corrente no ramo $\mathrm{km}$ do sistema; $f^{\max }$ é o fluxo máximo de corrente em cada ramo $\mathrm{km}$ do sistema; $V$ é a tensão calculada em cada seção do sistema; $V^{\text {min }}$ e $V^{\text {max }}$ são os limites mínimo e máximo de tensão em cada seção do sistema, respectivamente.

De acordo com as restrições, a equação (2) refere-se à capacidade de atendimento às cargas pelas subestações. A restrição dada pela inequação (3) avalia os fluxos de corrente nos ramais do sistema. A restrição dada pela inequação (4) avalia as tensões das seções do sistema. A restrição dada pela equação (5) representa o estado em que se encontra a seção da rede afetada pela falta após a restauração (ligada ou desligada do sistema). A restrição dada pela equação (6) garante que o sistema se mantenha com topologia radial após o estado restaurativo.

\section{Técnica de Solução}

Para a solução do PRSDEE é proposto um algoritmo BVNS [2], com uma estrutura de vizinhança e procedimento de busca local para promover mudanças na estrutura de vizinhança, baseada na técnica RNP. A RNP realiza podas no sistema através de três operadores [5]: PAO (Preserve Ancestor Operator), CAO (Change Ancestor Operator). Nesse trabalho, os operadores $\mathrm{PAO}$ e CAO são utilizados para gerar as estruturas de vizinhança do BVNS e o operador PAO é utilizado no procedimento de busca local na estrutura de vizinhança.

No algoritmo BVNS, deve-se escolher $k$ estruturas de vizinhança. O processo se inicia com uma solução $x \in X$ na primeira vizinhança $N_{1}(x)$. Em seguida, escolhe-se de forma aleatória um vizinho $x$ ' de $x$ em $N_{1}(x)$. A partir de $x$ 'é iniciado um processo de busca local para encontrar um ótimo local $x "$. Nesse contexto podem acontecer três casos: (1) se $x "=x$ significa que $x$ já era o ótimo local da vizinhança e, portanto, deve-se mudar para outro nível de vizinhança $\left(N_{2}(x)\right.$ neste caso); (2) se $x$ " é de pior qualidade que $x$ então foi encontrado um ótimo local de pior qualidade que a incumbente $x$ e também deve-se mudar de vizinhança; (3) se $x$ " é de melhor qualidade que $x$, significa que foi encontrada uma solução melhor que a incumbente e, portanto, deve-se atualizar a incumbente e reiniciar a busca permanecendo na vizinhança $N_{1}(x)$. Em qualquer iteração do processo, sempre que a busca local encontra uma nova incumbente volta-se para a vizinhança $N_{1}(x)$ e sempre que a busca local encontra uma solução de igual ou pior qualidade que a incumbente então passa-se para uma vizinhança maior. A estratégia do algoritmo BVNS e a escolha aleatória do vizinho $x$ evita o processo de ciclagem e permite encontrar ótimos locais distantes da solução incumbente. Caso a última vizinhança for alcançada sem que seja encontrada uma solução melhor que a incumbente, a busca é iniciada novamente na primeira vizinhança $N_{1}(x)$ até que uma condição de parada seja cumprida.

\subsection{Configuração inicial}

A configuração inicial é gerada através de uma heurística simples que consiste em religar às seções desenergizadas (exceto a seção da falta) através de seções vizinhas energizadas, sendo essa escolha de forma aleatória. Neste caso, todas as seções desenergizadas (exceto a seção da falta) são religadas por outra seção energizada, mesmo que a configuração topológica obtida não constitua uma solução factível.

\subsection{Estrutura de vizinhança e sistema de codificação}

A estrutura de vizinhança do algoritmo BVNS é baseada na quantidade de podas feitas pelos operadores da RNP, PAO e CAO, entre alimentadores que possuem seções afetadas pela falta e 
seus vizinhos, e assim sucessivamente, da seguinte forma:

- Vizinhança $N_{1}$ : realiza 1 poda no sistema entre alimentadores;

- Vizinhança $N_{2}$ : realiza 2 podas no sistema entre alimentadores;

- Vizinhança $N_{k}$ : realiza $k$ podas no sistema entre alimentadores.

Para essa estrutura de vizinhança são feitas as seguintes considerações:

1. Escolha do operador: as chances de escolha do operador PAO é de $70 \%$ e do operador CAO de $30 \%$. As chances de podas utilizando o operador CAO é menor do que do operador PAO para evitar muitas alterações bruscas na topologia da rede e os valores foram adotados baseados nas simulações;

2. Número máximo de podas $\left(k_{\max }\right)$ : é definido pela quantidade máxima de possibilidades de ramais de ligação (chaves NA) entre os alimentadores. O valor de $k_{\max }$ pode ser diferente para cada falta.

A Figura 1 ilustra um exemplo do sistema de codificação com a aplicação do operador PAO através da alteração do estado das chaves $c h_{12}$ e $c h_{9}$. O operador PAO realiza alterações simples na rede com a abertura e fechamento entre chaves de manobra adjacente, sendo essa escolha de forma aleatória.
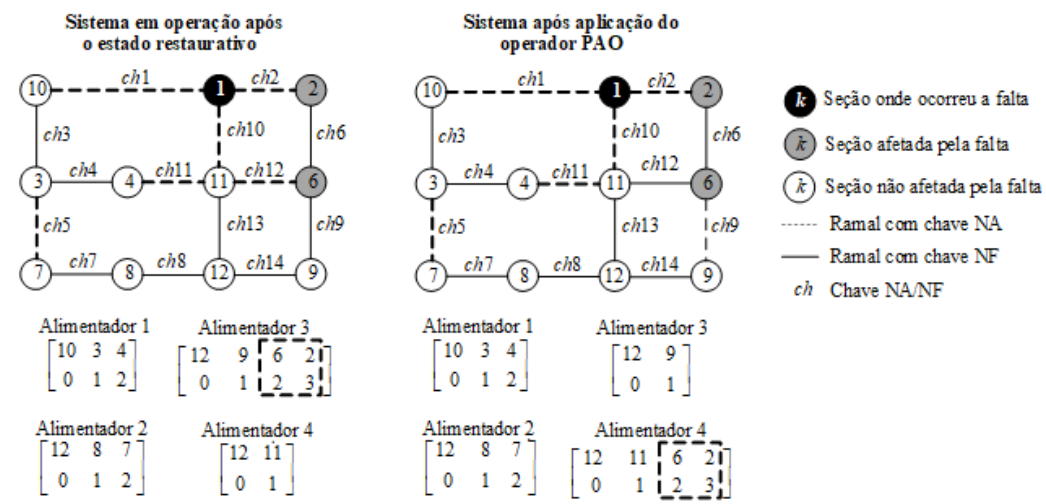

Figura 1: Sistema de codificação com aplicação do operador PAO

A Figura 2 ilustra o exemplo do sistema de codificação com a aplicação do operador CAO através da alteração do estado das chaves $c h_{12}$ e $c h_{14}$. O operador CAO realiza podas com alterações mais complexas na topologia da rede, através da abertura e fechamento de chaves em ramos quaisquer que permitem a conexão entre alimentadores vizinhos de forma que a nova topologia continue radial.

\subsection{Procedimento de busca local}

O procedimento de busca local, realizado para mudança na estrutura de vizinhança, é feito através da aplicação somente do operador PAO. Neste caso, as podas realizadas por esse operador, para alterar as estruturas topológicas da rede, ocorrem somente nas seções afetadas pela falta através da abertura e fechamento de chaves de manobras, sendo essa escolha de forma aleatória.

Assim, um vizinho na busca local seria uma nova topologia diferente da topologia corrente no que se refere ao estado das chaves de manobras ligadas a uma das seções afetada pela falta. 


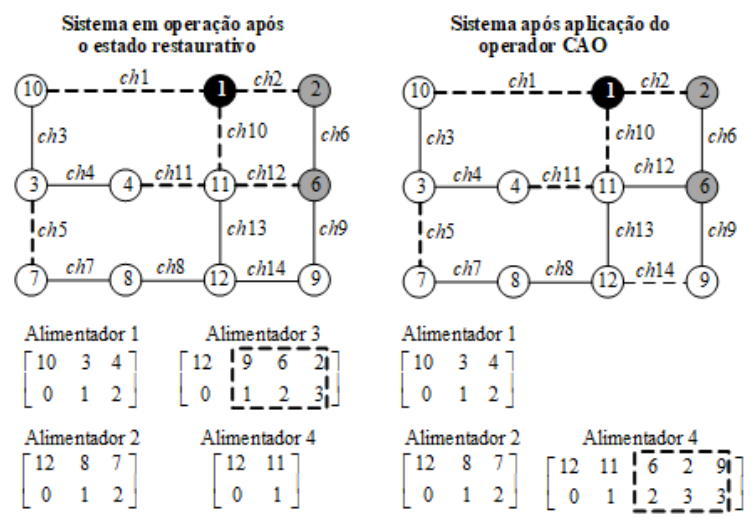

Figura 2: Sistema de codificação com aplicação do operador CAO

Para exemplificar o procedimento de busca local, vamos analisar os vizinhos da seção 6 (seção afetada pela falta) da Figura 3. Neste caso, tem-se a possibilidade de três vizinhos para a seção 6 , escolhidos de forma aleatória, sendo dois obtidos através do operador PAO da seguinte maneira:

- Vizinho 1: abertura da chave $c h_{9}$ e fechamento da chave $c h_{12}$;

- Vizinho 2: abertura da chave $c h_{9}$ e fechamento da chave $c h_{17}$;

- Vizinho 3: abertura da chave $c_{9}$ (seções 2 e 6 ficariam desligadas).

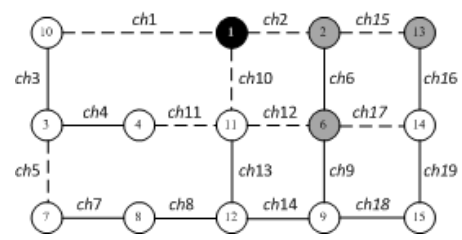

Figura 3: Exemplo de procedimento de busca local

\subsection{Avaliação das soluções}

Cada proposta de solução é avaliada através da função objetivo dada pela equação (1), sujeita às restrições impostas ao problema conforme as equações (2) a (6). Caso alguma dessas restrições sejam violadas a solução é penalizada.

\subsection{Critério de parada}

O critério de parada adotado para o algoritmo BVNS é o número máximo de iterações, determinado de acordo com o tamanho do PRSDEE.

\section{Resultados}

São apresentados resultados de testes para um sistema de 54 seções, sendo 3 subestações e 51 seções de cargas. A Figura 4 ilustra o sistema teste. Os dados do sistema teste podem ser 
encontrados em [4]. A Tabela 1 ilustra as seções interrompidas pela falta, bem como as seções afetadas pela interrupção. A Tabela 2 ilustra os dados de custos e parâmetros utilizados nas simulações. Ressalta-se que os custos referentes às violações das restrições foram ajustados durante as simulações. A Tabela 3 ilustra a proposta de restauração para as faltas com o respectivo custo de restauração.

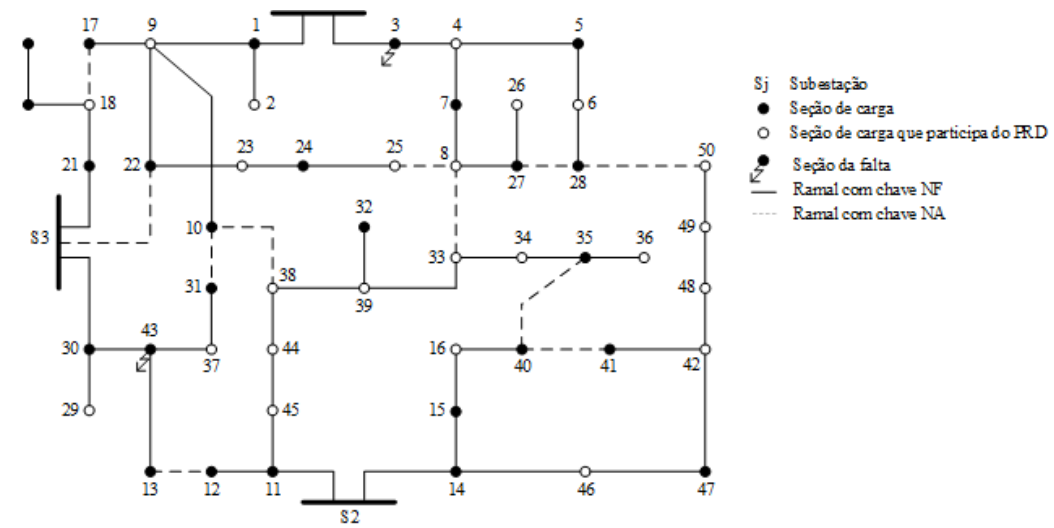

Figura 4: Sistema teste

Tabela 1: Dados das seções sob falta.

\begin{tabular}{cc}
\hline Seção da falta & Seções afetadas pela falta \\
\hline 3 & $4-5-6-7-8-26-27-28$ \\
43 & $13-31-37$ \\
\hline
\end{tabular}

Tabela 2: Dados de simulações.

\begin{tabular}{cccc}
\hline Custos & & Tensão \\
\hline$R_{C D}$ & $1.57 \$ / \mathrm{kW}$ & $V^{\min }$ & $0.95 \mathrm{pu}$ \\
$R_{P V}$ & $324.00 \$ /$ violação & $V^{\max }$ & $1.00 \mathrm{pu}$ \\
$R_{P f}$ & $445.00 \$ /$ violação & & \\
$R_{P S}$ & $445.00 \$ /$ violação & & \\
\hline
\end{tabular}

De acordo com a solução obtida pelo algoritmo BVNS, para que o sistema possa operar dentro dos limites operacionais do sistema elétrico, além das seções 3 e 43 onde ocorreram a falta, as seções 4, 5, 6, 26 e 27 não foram restabelecidas no processo de restauração, obtendo assim um custo de energia não distribuída no valor de 8,583.17 (\$), conforme ilustra a Tabela 3. Para este caso, o tempo de simulação foi de 2,1 segundos.

Comparando os resultados referente a falta ocorrida na seção 3 obtidos pelo algoritmo BVNS com os resultados obtidos em [4], o qual utiliza como técnica de solução um algoritmo Tabu Search, o algoritmo BVNS conseguiu restabelecer o mesmo potencial de cargas que o algoritmo Tabu Search, porém com um tempo de processamento menor, sendo que o tempo de processamento do algoritmo Tabu Search foi de 3,12 segundos. Neste caso, acredita-se que a utilização do algoritmo BVNS será de grande vantagem na reolução do PRSDEE, quando se tratar de sistemas de grande porte. 
Tabela 3: Proposta de restauração.

\begin{tabular}{ccc}
\hline Proposta & Solução & Custo $\left(C_{E N D}\right)$ \\
\hline Seções restauradas & $7-8-13-28-31-37$ & \\
Seções não restauradas & $4-5-6-26-27$ & $8,583.17(\$)$ \\
Ramais com chaves NF alteradas & $\mathrm{S} 1-3 ; 3-4 ; 4-7 ; 6-28 ; 8-27 ; 9-17 ;$ & \\
& $9-18 ; 30-43 ; 43 ; 13 ; 43-37$ & \\
Ramais com chaves NA alteradas & $\mathrm{S} 3-22 ; 8-25 ; 10-31 ; 12-13 ;$ & \\
& $17-18 ; 28-50$ & \\
\hline
\end{tabular}

\section{Conclusões}

Este trabalho apresentou um modelo de restauração de redes de distribuição utilizando a técnica de otimização conhecida como algoritmo BVNS (Basic Variable Neighborhood Search). O algoritmo BVNS, cuja estrutura de vizinhança utiliza a aplicação da técnica RNP, mostrou ser eficiente ao efetuar buscas locais com mudanças de estruturas de vizinhança em torno do ótimo local, e restabelecer o sistema de forma otimizada com um baixo tempo de simulação. O modelo de restauração utilizando o algoritmo BVNS é útil, confiável e de fácil aplicação, podendo ser utilizado pelas concessionárias de energia elétrica na obtenção de resultados satisfatórios para a restauração do sistema elétrico, fazendo o remanejamento adequado de seções entre alimentadores, buscando restaurar o máximo possível de seções de cargas obedecendo as restrições operacionais e técnicas da rede elétrica.

\section{Referências}

[1] Abdelaziz, A. Y., Mohamed, F. M., Mekhamer, S. F. and Badr, M. A. L. Distribution system reconfiguration using a modified Tabu Search algorithm, Electric Power System Research, 80:943-953, 2010.

[2] Hansen, P. and Mladenovic, N. Variable neighborhood search: principles and applications, European Journal of Operational Research, 130:449-467, 2001.

[3] Morelato, A. L. and Monticelli, A. J. Heuristic search approach to distribution system restoration, IEEE Transactions on Power Delivery, 4:2235-2241, 1989.

[4] Pereira Júnior, B. R., Cossi, A. M. e Mantovani, J. R. S. Proposta de uma metodologia baseada em busca tabu para restaura, c $^{\sim}$ ao automática de sistemas de distribuição de energia elétrica, Congresso Brasileiro de Automática, pages 1204-1211, 2012. ISBN: 978-85-8001-069-5.

[5] Santos, A., Delbem, A., London, J. and Bretas, N. Node-depth encoding and multiobjective evolutionary algorithm applied to large-scale distribution system reconfiguration, IEEE Transactions on Power Delivery, 25:1254-1265, 2010.

[6] Toune, S., Fudo, H., Genji, T., Fukuyama, Y. and Nakanishi, Y. Comparative study of modern heuristic algorithms to service restoration in distribution systems, IEEE Transactions on Power Delivery, 17:173-181, 2002.

[7] Vargas, R., Mathias Neto, W. P., Silva, L. G. W. and Mantovani, J. R. S. Automatic Restoration of Active Distribution Networks Based on Tabu Search Specialized Algorithm, Conference: IEEE PES Innovative Smart Grid Technologies Latin America (ISGT LATAM), pages 411-416, 2015. DOI:10.1109/ISGT-LA.2015.7381190. 